\title{
No Slip CMB
}

\author{
Micah Brush ${ }^{1}$, Eric V. Linder ${ }^{1,2}$, Miguel Zumalacárregui ${ }^{1,3}$ \\ ${ }^{1}$ Berkeley Center for Cosmological Physics \& Berkeley Lab, \\ University of California, Berkeley, CA 94720, USA \\ ${ }^{2}$ Energetic Cosmos Laboratory, Nazarbayev University, Astana, Kazakhstan 010000 \\ ${ }^{3}$ Institut de Physique Théorique, Université Paris Saclay CEA, CNRS, 91191 Gif-sur-Yvette, France
}

(Dated: October 31, 2018)

\begin{abstract}
No Slip Gravity is a simple modified gravity theory with only one free function and the interesting characteristic that - unlike many modified gravity theories - it suppresses growth. This allows it to fit current redshift space distortion and $\sigma_{8}$ mass fluctuation amplitude data better than $\Lambda$ CDM in general relativity, while retaining a $\Lambda$ CDM background expansion and hence distances. Since it has no gravitational slip it alters equally CMB lensing and matter density growth, and in addition affects the CMB gravitational wave B-mode polarization power spectrum. We investigate and compute the effects of No Slip Gravity for CMB lensing and B-modes, and present a simple analytic approximation. Using a Monte Carlo analysis, we place constraints on the theory from current CMB data.
\end{abstract}

\section{INTRODUCTION}

Acceleration of the cosmic expansion may be caused by a cosmological constant or scalar field, or a modification of the laws of gravity. Once the expansion history is given, general relativity implies that the growth of cosmic structure is determined, but modified gravity allows deviations to exist and so offers further signatures. If cosmic growth is indeed lower than expected from general relativity plus a background expansion near a cosmological constant and cold dark matter $(\Lambda \mathrm{CDM})$ universe, as several current data sets from redshift space distortions and weak gravitational lensing may imply [1-5], then modified gravity is an interesting possibility to examine.

Generally scalar-tensor theories of gravity lead to an enhancement of growth, however, due to the scalar field adding to the gravitational strength. A theory with a strong braiding between the scalar and tensor sectors can counteract this, and even weaken gravity and growth. One interesting example of such a theory is No Slip Gravity [6]. This also has the useful property that there is only one (out of a generic four) modified gravity function (formally, property functions [7]) that must be specified. Thus it can be a fairly predictive theory.

Since this one free function, which can be regarded as the running of the Planck mass, also affects the propagation of gravitational waves (even though their speed is fixed to the speed of light), No Slip Gravity is of particular interest for experiments seeking primordial gravitational waves signatures from inflation in the cosmic microwave background (CMB) B-mode polarization power spectrum. In addition, [6] showed that the modified propagation of gravitational waves from late universe sources such as could be detected by ground and space based gravitational wave interferometers must be directly related to an offset in the distances to a source derived from gravitational wave emission vs from photons - and to structure growth - a testable prediction.

Finally, the suppression of cosmic structure growth that would be seen in galaxy clustering surveys should also be directly related to a dilution in the CMB lensing power spectrum, due to the no slip nature of the theory, i.e. that the gravitational strength for influencing matter and light are equal. This implies the small angular scale part of the CMB B-mode polarization power spectrum, dominated by lensing, should be affected.

This tight interrelation between many observables, whose measurements will be increasingly precise with the next generation of galaxy clustering, CMB, weak lensing, and gravitational wave experiments, makes No Slip Gravity well worth testing. Here we used hi_class, a Boltzmann code enhanced to include modified gravity property functions in the equations, to compute the CMB power spectra, especially lensing and B-modes, and investigate the signatures of No Slip Gravity.

In Section II we briefly review modified gravity property functions, No Slip Gravity, and the hi_class code. We evaluate the CMB B-mode polarization power spectrum in Sec. III and elucidate the physical effects on the tensor (gravitational wave) part and the lensing part. Section IV investigates in greater detail the CMB lensing power spectrum and gives a simple analytic approximation for the effects. We carry out a Markov Chain Monte Carlo analysis of the constraints from current CMB data in Sec. V, and discuss the results and conclude in Sec. VI.

\section{METHODS FOR THEORY AND CODE}

The property functions of modified gravity [7] are an effective field theory like approach to characterizing deviations from general relativity, with each property function closely related to a particularly characteristic. In standard Horndeski theory, describing scalar-tensor gravity that yields second order equations of motion, four property functions enter: $\alpha_{K}$ giving the kinetic stiffness of the scalar field fluctuations, $\alpha_{B}$ the kinetic mixing (or braiding) between the scalar field and the metric, $\alpha_{M}$ the running of the effective Planck mass (on the cosmological background), and $\alpha_{T}$ the deviation of the speed of tensor 
perturbation (gravitational wave) propagation from the speed of light. Each is a dimensionless function of time, or cosmic scale factor, and their vanishing indicates that gravity is general relativity.

Certain subclasses of Horndeski gravity have relations between the property functions, with the two main examples being $f(R)$ gravity, Brans-Dicke, or chameleon theories where $\alpha_{B}=-\alpha_{M}$ and No Slip Gravity where $\alpha_{B}=-2 \alpha_{M}$. It is the condition

$$
\alpha_{B}=-2 \alpha_{M} \quad \text { (No Slip Gravity) }
$$

that gives the physical characteristic of no slip, i.e. the metric potentials are equivalent (neglecting matter anisotropic stress) and so the gravitational coupling strengths $G_{\text {matter }}$ and $G_{\text {light }}$ (generalizations of Newton's constant) that enter the modified Poisson equations for density perturbations and light propagation are equal.

As mentioned in the Introduction, No Slip Gravity has some interesting physical properties in addition to its mathematical simplicity, with an intriguing suppression of growth. It can also readily be made ghost free, satisfying $\alpha_{K}+(3 / 2) \alpha_{B}^{2} \geq 0$ (see the discussion of $\alpha_{K}$ below), and has a remarkably simple stability condition,

$$
\frac{d^{2} \ln M_{\star}^{2}}{d t^{2}} \leq 0 \quad \text { or } \quad\left(H \alpha_{M}\right)^{\cdot} \leq 0,
$$

where $M_{\star}$ is the effective Planck mass, $H$ the Hubble parameter, and dot denoting a time derivative. Indeed, for certain parametrizations of $\alpha_{M}(a)$, No Slip Gravity is an exemplar, a "maximally stable" model [8].

We follow the parametrizations for $\alpha_{M}(a)$ given in [6], that were stable and ghost free. While we tested both the $M_{\star}$ and $\alpha_{M}$ parametrizations given there, we found them substantially equivalent and so we present the results for

$$
\begin{aligned}
\alpha_{M}(a) & =c_{M}\left(1-\tanh ^{2}\left[(\tau / 2) \ln \left(a / a_{t}\right)\right]\right) \\
& =\frac{4 c_{M}\left(a / a_{t}\right)^{\tau}}{\left[\left(a / a_{t}\right)^{\tau}+1\right]^{2}} .
\end{aligned}
$$

Note that $\alpha_{M}$ approaches zero in the past (general relativity at high redshift) and in the future (consistent with the de Sitter asymptote of $\Lambda \mathrm{CDM}$ ), and has a maximum value $c_{M}$ at $a=a_{t}$. The stability condition is just $\tau \leq 3 / 2$. We set fiducial values $a_{t}=0.5, \tau=1$, and $M_{\star, \text { ini }}^{2}=m_{p}^{2}$, which are used for the rest of the paper.

We set $\alpha_{T}=0$, since this is the most straightforward interpretation of the GW170817/GRB170817A gravitational wave and electromagnetic counterpart observations [9]. The kineticity $\alpha_{K}$ generally has little effect on observational quantities on subhorizon scales (see the discussion below). We take the background expansion history to be that of $\Lambda \mathrm{CDM}$ so that we can compare the effects on the CMB and growth purely due to the gravitational deviations from general relativity.

The model was introduced into the hi_class code $[10,11]$ by imposing the No Slip Gravity condition $\alpha_{B}=-2 \alpha_{M}$, with the specific time dependence given by Eq. (3). The effective Planck mass $M_{\star}$ is obtained from the definition $\alpha_{M} \equiv d \ln M_{\star}^{2} / d \ln a$. These modeldependent specifications are included in the background module of hi_class and are read automatically by the perturbations module to solve the linearly-perturbed, modified Einstein equations for Horndeski gravity. A public version of hi_class including these modifications is available at https://github.com/micbru/hi_class_ public. It can also be used to specify $A_{\text {lens }}$ (as discussed in Secs. IVand V).

To test the influence of $\alpha_{K}$, and in particular to separate out its effect in No Slip Gravity, we studied the results for several different values approaching $\alpha_{K}=0$. One cannot generally set $\alpha_{K}=0$ identically because the inverse of the kinetic term $\alpha=\alpha_{K}+(3 / 2) \alpha_{B}^{2}$ diverges in the limit in which $\alpha_{B} \rightarrow 0$, as happens at early times. This divergence produces very rapid oscillations of the scalar field perturbations that cannot be resolved numerically. Furthermore, these oscillations have no impact on the predictions in the limit in which $\Omega_{\phi}, \alpha_{B}, \alpha_{M}$ are all negligible, which is satisfied at early times in the models we are considering.

To overcome these numerical problems at early times we add a constant value to $\alpha_{K}$ through the kineticity_safe_smg parameter in hi_class. We tested that this value is small enough to produce a negligible deviation in the $\mathrm{CMB}$ spectra: Figure 1 compares the $\mathrm{CMB}$ temperature power spectrum $C_{\ell}^{T T}$ and the matter density perturbation power spectrum $P(k)$ for different values of this parameter while holding the other parameters fixed. This value is several orders of magnitude below the sensitivity of even next-generation experiments [12].

Indeed, $\alpha_{K}$ has little influence on subhorizon scales (wavenumbers $k \gtrsim 10^{-3} h / \mathrm{Mpc}$ ). For scales near the horizon, as long as $\alpha_{K} \lesssim 10^{-3}$ the effect on the power spectra is negligible. We set it to $10^{-5}$ for the rest of the computations.

To ensure that small kineticity_safe_smg works as expected, we also made $\alpha_{K}$ near zero by setting $\alpha_{K}=$ $10^{-4} \alpha_{M}$. There was no discernible difference between these two methods for setting $\alpha_{K}=0$ and we believe them to be essentially equivalent at the level of precision of both modified gravity Boltzmann codes and current data [13].

\section{CMB B-MODE POLARIZATION}

An observable of great interest for future surveys is the $\mathrm{B}$ mode polarization power spectrum of the CMB. This has two main contributions: at large angular scales, low multipoles $\ell$, the $\mathrm{B}$ modes arise from the primordial gravitational waves generated by inflation in the early universe. Their amplitude is a direct measure of the inflationary energy scale. Furthermore, they test the theory of gravity because they involve the propagation of gravitational waves over cosmic distances. At small angular scales, high multipoles, the dominant B mode 

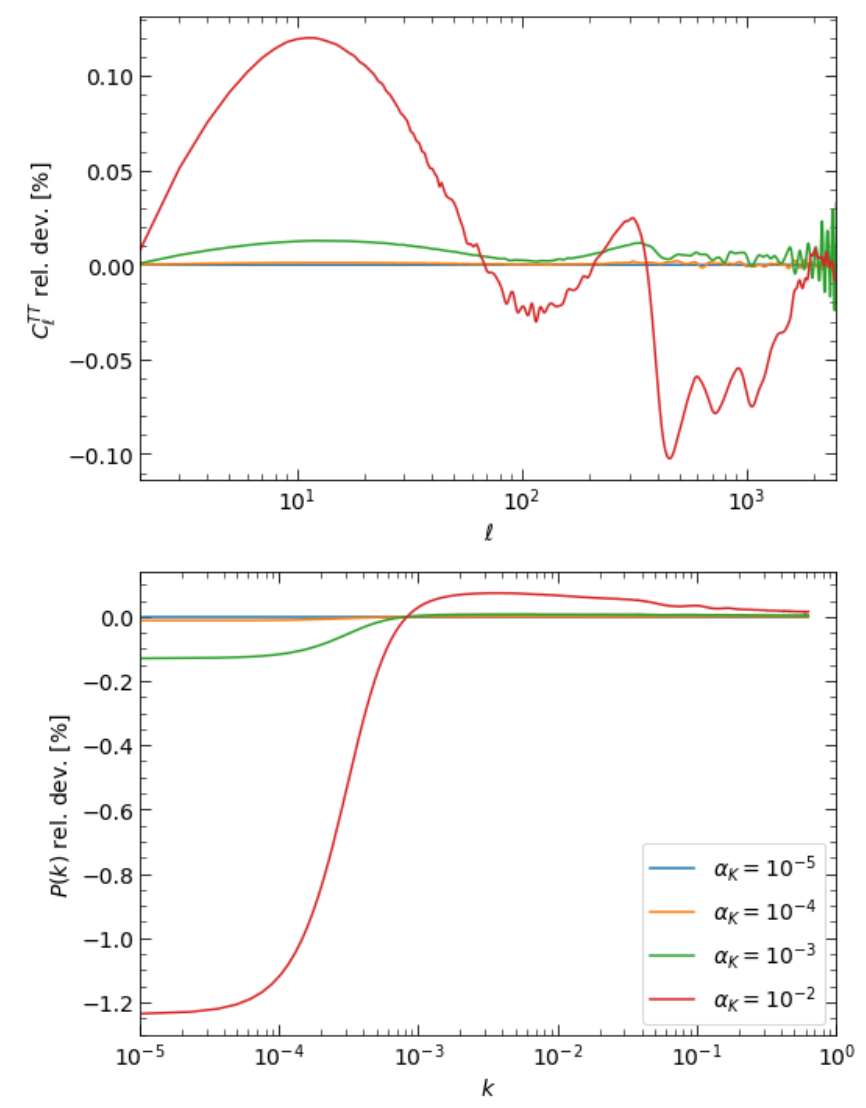

FIG. 1. The CMB temperature power spectrum $C_{\ell}^{T T}$ and the matter density perturbation power spectrum $P(k)$ compared with different values of constant $\alpha_{K}$ relative to $\alpha_{K}=10^{-5}$. The deviations are well under a percent, with only the matter power spectrum showing larger deviations for $\alpha_{K} \gtrsim 10^{-2}$ on near horizon scales. $c_{M}$ is held fixed at 0.1 .

contribution comes from gravitational lensing of the $\mathrm{E}$ mode polarization and so does not substantially involve the tensor perturbations. However, the strength of gravitational lensing depends on the matter density perturbation power spectrum, and hence the growth of cosmic structure, and also the gravitational coupling strength for light propagation. Thus it tests both structure growth and gravity.

For primordial gravitational waves, the main signatures are a reionization bump at $\ell \lesssim 10$ and a recombination bump at $\ell \approx 100$ in the $B$ mode spectrum. Since the gravitational wave propagation speed is unchanged from general relativity ( scale of the bumps are unchanged by the modified gravity, just the amplitude. For primordial tensor to scalar ratio $r \lesssim 0.01$, the recombination bump is mostly hidden beneath the late time lensing contribution. Figure 2 shows the tensor only (no lensing) contributions to the $\mathrm{B}$ modes with $r=0.1$ for different amplitudes $c_{M}$, relative to the general relativity case.

The manner in which $\alpha_{M}$ enters into gravitational wave propagation is through the friction term in the wave

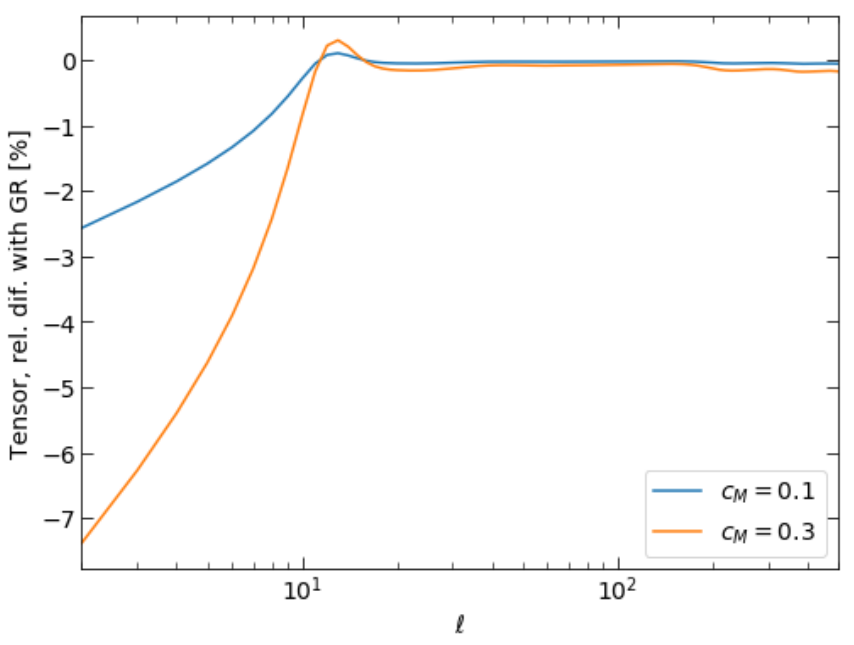

FIG. 2. The primordial CMB B modes give signatures of modified gravity, even if the gravitational wave speed is the speed of light. The relative deviation of the tensor power spectra $C_{\ell}^{B B}$ (without lensing) for No Slip Gravity models with $c_{M}=0.1$ and 0.3 is shown with respect to general relativity $\left(c_{M}=0\right)$.

propagation (see e.g. $[14,15])$. Positive $\alpha_{M}$ damps the gravitational wave amplitude. This can also be thought of as increasing the luminosity distance of the gravitational wave source $[16,17]$. Thus the $\mathrm{B}$ mode power is reduced. The rule of thumb for this No Slip Gravity model is that the reionization bump is reduced by

$$
\Delta P_{B B} / P_{B B} \approx 20 c_{M} \% \text {. }
$$

In contrast, the recombination bump is quite insensitive to $c_{M}$ in this model. This is because $\alpha_{M}$ is time dependent and is much smaller at $z \approx 1000$ than at $z \approx 6$. Note that [8] used a model where $\alpha_{M}=c_{M} a$ and in their Figure 4 found a comparable effect on the B mode reionization bump, when accounting for the time dependence and different value of $c_{M}$.

Since the reionization bump amplitude is proportional to the tensor-scalar ratio $r$, there is a degeneracy between the inflationary and modified gravity signatures if we only detect the reionization bump. The bias is

$$
\delta r / r \approx 0.2 c_{M}
$$

Since we expect $c_{M} \approx 0.03$ (at least if it is connected with the suppression of growth at the current data level), this is less than a $1 \%$ bias. If $r=0.01$, then even for Stage 4 CMB experiments the bias will not be significant. Moreover, the higher multipole B mode spectrum will have a separate modified gravity signature that could break this degeneracy (though it may involve other covariances, as we will see in Sec. V).

We now turn to the overall B mode spectrum, including both the tensor contributions (with $r=0.1$ to make the recombination bump clearly visible) and lensing contributions. Figure 3 shows the full B mode spectrum where 


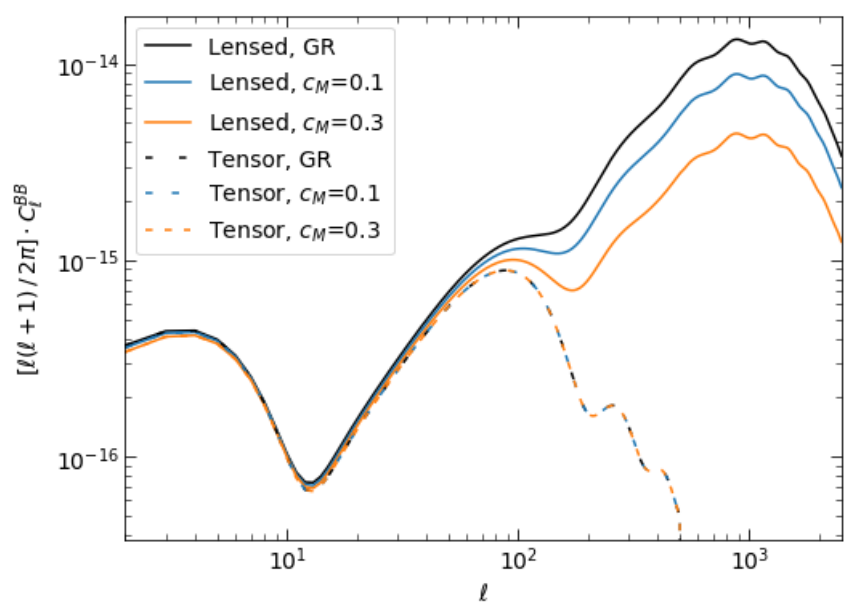

FIG. 3. CMB B mode power spectra including both lensing and primordial tensor (with $r=0.1$ ) contributions are plotted for the No Slip Gravity models with $c_{M}=0.1$ and 0.3 , and general relativity $\left(c_{M}=0\right)$. The dotted curves show the same models but for the tensor contributions alone.

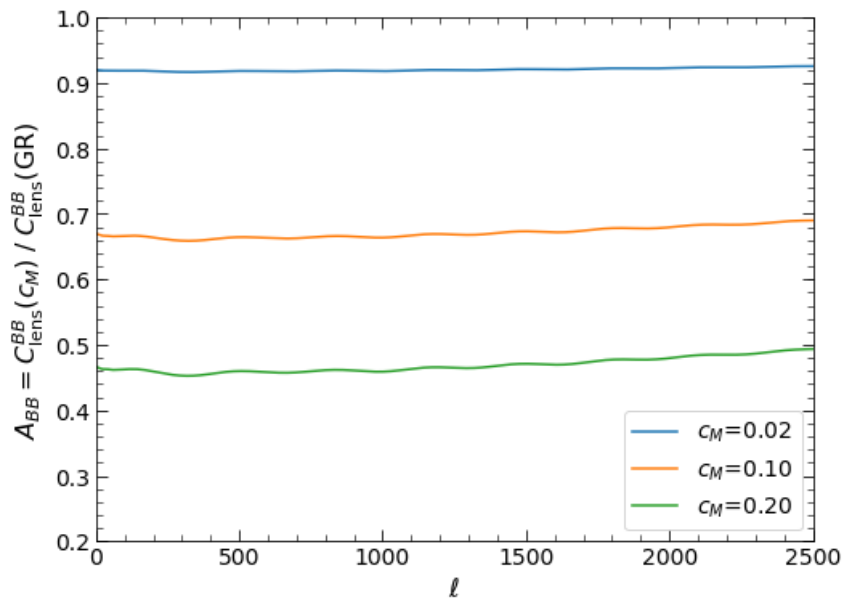

FIG. 4. Ratios of the lensed B modes (no tensor contribution) in No Slip Gravity for the indicated values of $c_{M}$ relative to GR.

we can see the two primordial bumps plus the lensing peak at $\ell \approx 1000$. The effect of $\alpha_{M}$ on the lensing power is much more pronounced there.

Since a significant effect is visible in the high multipoles from the CMB lensing, we now focus on the CMB B mode lensing by setting $r=0$. Figure 4 shows the ratio of the scalar lensed $C_{\ell}^{B B}$ between No Slip Gravity and general relativity for various $c_{M}$. Increasing $\alpha_{M}$ suppresses the power of the lensing $B$ modes as it weakens the growth and matter power spectrum.

The lensing B modes exhibit a substantially scale independent dilution, given approximately by

$$
A_{B B} \equiv \frac{C_{\text {lens }}^{B B}\left(c_{M}\right)}{C_{\text {lens }}^{B B}(G R)} \approx 1-4 c_{M}
$$

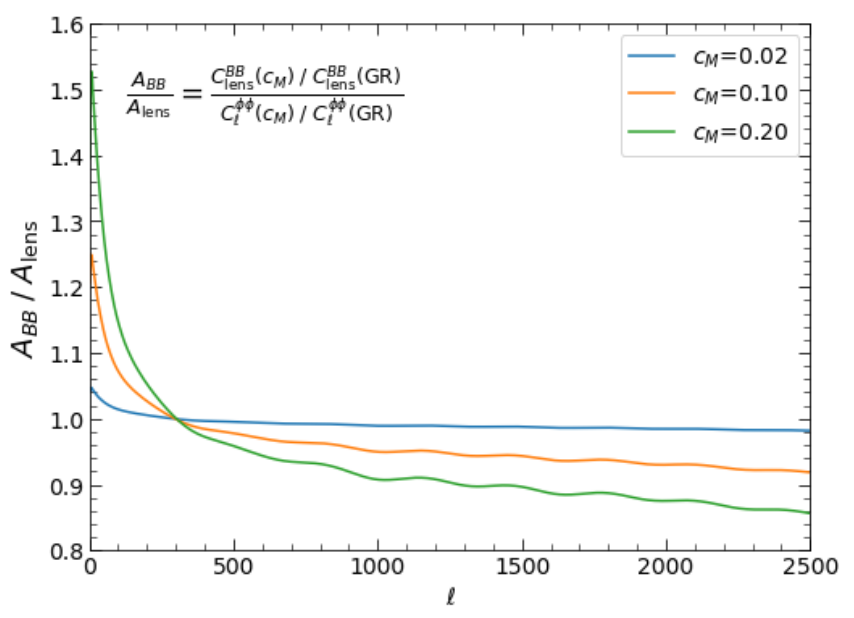

FIG. 5. Ratio of ratios: the lensing B mode power spectrum relative to the CMB lensing power spectrum is compared between the No Slip Gravity cases and the general relativity prediction, or equivalently the ratio of lensing B modes in No Slip Gravity relative to general relativity $\left(A_{B B}\right)$ is compared to the ratio of lensing power in No Slip Gravity relative to general relativity $\left(A_{\text {lens }}\right)$.

for $c_{M} \ll 1$. We explore the magnitude of $A_{B B}$ further in the next section.

Since the lensing B modes arise from gravitational lensing (affected by the late time modified gravity) of the primordial E modes (unaffected by the late time modified gravity), it is useful to directly compare the CMB lensing deflection power spectrum. The ratio of $C_{L}^{\phi \phi}\left(c_{M}\right) / C_{L}^{\phi \phi}(G R)$ is called $A_{\text {lens }}$ and is not identical to $A_{B B}$ due to a convolution going from $C^{\phi \phi}$ to $C_{\text {lens }}^{B B}$. Figure 5 illustrates how the ratio behaves.

In this case scale dependence is evident. There is an enhancement in the ratio $A_{B B} / A_{\text {lens }}$ at low $\ell$ and a dilution at high $\ell$. We explore the origin of this in the next section.

\section{CMB LENSING POWER SPECTRUM}

To separate the effects seen in the previous section about the effects of No Slip Gravity on the B mode power spectrum (dominated by lensing) and the CMB lensing power spectrum itself, we now investigate the CMB lensing power spectrum. This comes from the power spectrum of the gravitational potential along the line of sight, $C_{\ell}^{\phi \phi}$.

Figure 6 plots $C_{\ell}^{\phi \phi}$ in No Slip Gravity for various $c_{M}$ as well as in general relativity. As expected, the gravitational potential power is diminished as structure growth is suppressed by increasing $c_{M}$. We have also studied the effect of varying $a_{t}$ and $\tau$ from their fiducial values, but found that the effects were fairly degenerate with changing $c_{M}$. The key quantity seems to be the integrated amplitude of the modified gravity deviation over the red- 


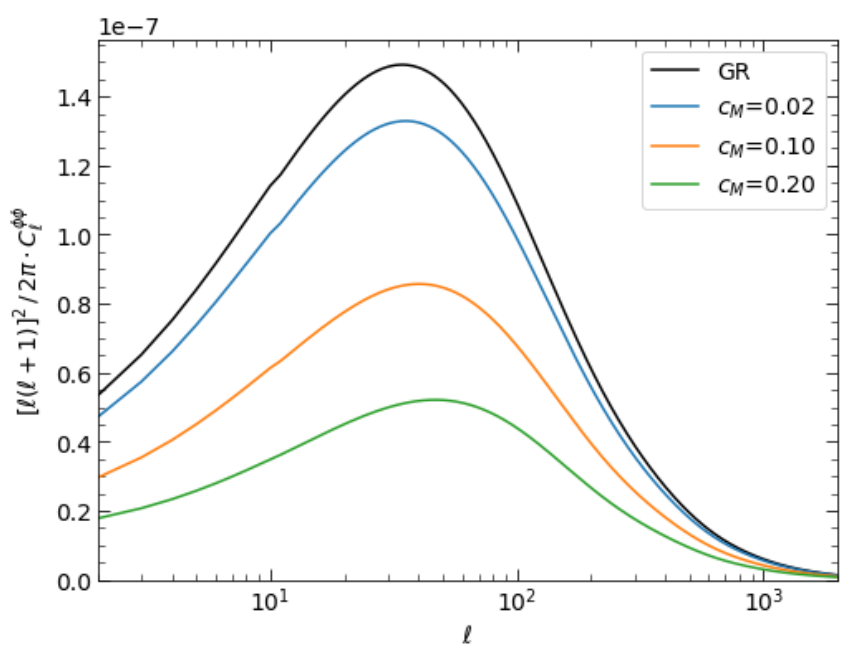

FIG. 6. The CMB lensing power spectra (or deflection angle power with the extra factors of $\ell$ ) are plotted for No Slip Gravity for various $c_{M}$ and for general relativity $\left(c_{M}=0\right)$.

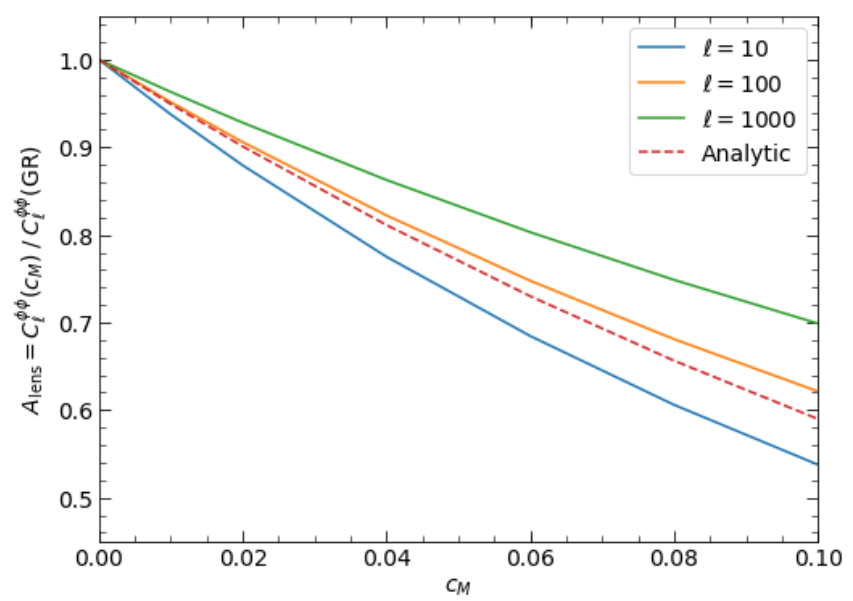

FIG. 7. The ratio of the CMB lensing power spectrum between No Slip Gravity and general relativity, as a function of modified gravity strength $c_{M}$, gives a clear idea of the physical growth suppression. Different solid color curves represent different scales $\ell$. The dashed curve is the approximate analytic result from Eq. 15.

shift range during which $\mathrm{CMB}$ lensing has the greatest effect, $z \approx 1-3$. We give an analytic justification for this numerical finding below.

To show the impact of No Slip Gravity more directly, and the scaling with $c_{M}$ as a function of scale, we plot the deviation of the lensing power $C_{\ell}^{\phi \phi}$ from the general relativity result as a function of $c_{M}$ for several multipoles $\ell$ in Fig. 7. $C_{\ell}^{\phi \phi}$ can exhibit significant deviations from general relativity, depending on $c_{M}$ and to a lesser extent $\ell$, i.e. there is some scale dependence.

We can explore the effect on the CMB lensing power spectrum in a heuristic, analytic manner. A key characteristic of No Slip Gravity is that matter and light feel the same gravitational strength. In terms of the modified Poisson equation gravitational strengths (dimensionless, i.e. in units of Newton's constant $G_{N}$ ),

$$
G_{\text {light }}=G_{\text {matter }}=\frac{m_{p}^{2}}{M_{\star}^{2}},
$$

where the first equality is an expression of no slip, and the second follows from the $\alpha_{B}=-2 \alpha_{M}$ relation in No Slip Gravity [6].

In illustrative terms, the CMB lensing power spectrum

$$
C_{\ell}^{\phi \phi} \sim\left[\nabla^{2}(\Phi+\Psi)\right]^{2} \sim G_{\text {light }}^{2}(\delta \rho / \rho)^{2},
$$

where $\Phi$ and $\Psi$ are the time-time and space-space metric potentials, $G_{\text {light }}$ is the modified gravitational strength in the relativistic Poisson equation

$$
\nabla^{2}(\Phi+\Psi)=8 \pi G_{N} G_{\text {light }}(\delta \rho / \rho),
$$

and $\delta \rho / \rho$ is the linear matter density contrast.

If we now take the ratio of $C_{\ell}^{\phi \phi}$ to that from general relativity $\Lambda \mathrm{CDM}$, we have

$$
\frac{C_{\ell}^{\phi \phi}}{C_{\ell}^{\phi \phi}(\mathrm{GR})} \approx G_{\text {light }}^{2}\left(1+2 \frac{\delta g}{g_{\mathrm{GR}}}\right),
$$

where $g=(\delta \rho / \rho) / a$ is the density perturbation growth factor and $\delta g$ is the (small) difference from the general relativity case. Since much of the contribution to CMB lensing occurs at redshift $z \approx 3$, we can use the modified gravity growth approximation of [18] that relates the early time modified growth to $3 / 5$ times the area under the modified gravitational strength function, Area $\sim \int d \ln a\left(G_{\text {matter }}-1\right)$. This is intended as a rough rule of thumb.

Putting this together, we find

$$
\begin{aligned}
\frac{C_{\ell}^{\phi \phi}}{C_{\ell}^{\phi \phi}(\mathrm{GR})} & \approx\left(\frac{m_{p}^{2}}{M_{\star}^{2}}\right)^{2}\left[1+\frac{6}{5} \text { Area }\right] \\
& \approx\left(\frac{m_{p}^{2}}{M_{\star}^{2}}\right)^{2}\left[1+\frac{6}{5} \int d \ln a\left(\frac{m_{p}^{2}}{M_{\star}^{2}}-1\right)\right]
\end{aligned}
$$

Finally we use the No Slip Gravity model expression for $M_{\star}^{2}[6]$,

$$
\frac{m_{p}^{2}}{M_{\star}^{2}}=e^{-\left(2 c_{M} / \tau\right)\left[1+\tanh \left((\tau / 2) \ln \left(a / a_{t}\right)\right]\right.},
$$

and evaluate it near the peak $a \approx a_{t}$, using a "full width half maximum" weighting on the integral to find

$$
\frac{C_{\ell}^{\phi \phi}\left(c_{M}\right)}{C_{\ell}^{\phi \phi}(\mathrm{GR})} \approx e^{-4 c_{M} / \tau}\left(1-\frac{6 c_{M}}{5}\right) .
$$

This is shown as the dashed "Analytic" curve in Fig. 7, and works surprisingly well at multipoles near the maximum of the lensing power spectrum. For small amplitudes $c_{M} \ll 1$ this gives a lensing power spectra ratio of 
$\approx 1-5 c_{M}$. Note that while this ratio is basically $A_{\text {lens }}$, in fact $A_{\text {lens }}$ is usually taken to be scale independent.

The lensing $\mathrm{B}$ mode power is a convolution of the lensing power spectrum with the $\mathrm{E}$ mode polarization power spectrum. The power $C_{\ell}^{B B}$ at some multipole actually arises from a range of multipoles in $C_{\ell^{\prime}}^{\phi \phi}$. This tends to weaken the scale dependence of $C_{\ell}^{B B} / C_{\text {lens }}^{B B}(\mathrm{GR})$ as seen in Fig. 4.

We can also gain intuition on Fig. 5. At low multipoles, the convolution prefers higher lensing multipoles, shifted up toward the peak of the lensing power spectrum, while at high multipoles it prefers somewhat lower lensing multipoles, again toward the peak. But from Fig. 7 we see that shifting low lensing multipoles to higher ones relaxes the dilution suffered by the lensing power, while shifting high lensing multipoles to lower ones increases the dilution. Thus, at low multipoles $A_{B B}>A_{\text {lens }}$ and at high multipoles $A_{B B}<A_{\text {lens }}$ as found in Fig. 5. Current uncertainties on $A_{B B}$ are large, with the POLARBEAR CMB experiment quoting $A_{B B}=0.69_{-0.25}^{+0.26}\left(\right.$ stat $\left._{-0.04}^{+0}\right)$ (inst \pm 0.04$)$ [19]. While POLARBEAR's estimate of $A_{\text {lens }}$ is slightly lower, Planck's much more precise value is $A_{\text {lens }}=1.180 \pm 0.065$ [20], i.e. $A_{B B}<A_{\text {lens }}$. However it is much too early to attribute any significance to this.

\section{CURRENT CONSTRAINTS}

To explore CMB constraints on No Slip Gravity, in the form of the effective $c_{M}$ amplitude of the modified gravity deviation (i.e. folding the $a_{t}$ and $\tau$ parameters into a single effective amplitude parameter) we run a Markov Chain Monte Carlo Analysis using the MontePython code [21, 22] interfaced with hi_class. Precision B mode data does not yet exist so we use only the Plik TT likelihoods from the Planck 2015 release [23]. The parameter $c_{M}$ is added to the six standard cosmology parameters, with a stability restriction $c_{M} \geq 0$.

We find that the $95 \%$ confidence upper limit on $c_{M}$ is 0.015. Such a tight limit arises from two effects. As we have seen, $c_{M}$ suppresses the lensing power spectrum, while Planck data prefer a higher than $\Lambda$ CDM strength of lensing, i.e. smoothed acoustic oscillations corresponding to $A_{\text {lens }}=1.18$ [20]. Secondly, $c_{M}$ gives a larger SachsWolfe effect on large angles (low multipoles), again in tension with what the data suggest. We can separate out these effects by looking at $\ell>30$ data vs $\ell<30$ data and find that each gives roughly the same $\Delta \chi^{2} \sim 10$ penalty to a value of $c_{M}=0.03$ with respect to general relativity $\left(c_{M}=0\right)$. Recall that conversely at least some large scale structure data prefer suppressed growth as discussed in [6].

Since Planck data prefer a noncanonical (i.e. $\Lambda \mathrm{CDM}$ plus general relativity) $A_{\text {lens }}$ with an unknown origin, we can try adding both $c_{M}$ and $A_{\text {lens }}$ to the standard six parameters. As we expect from Fig. 7, the two can compensate for each other over a broad range of multipoles

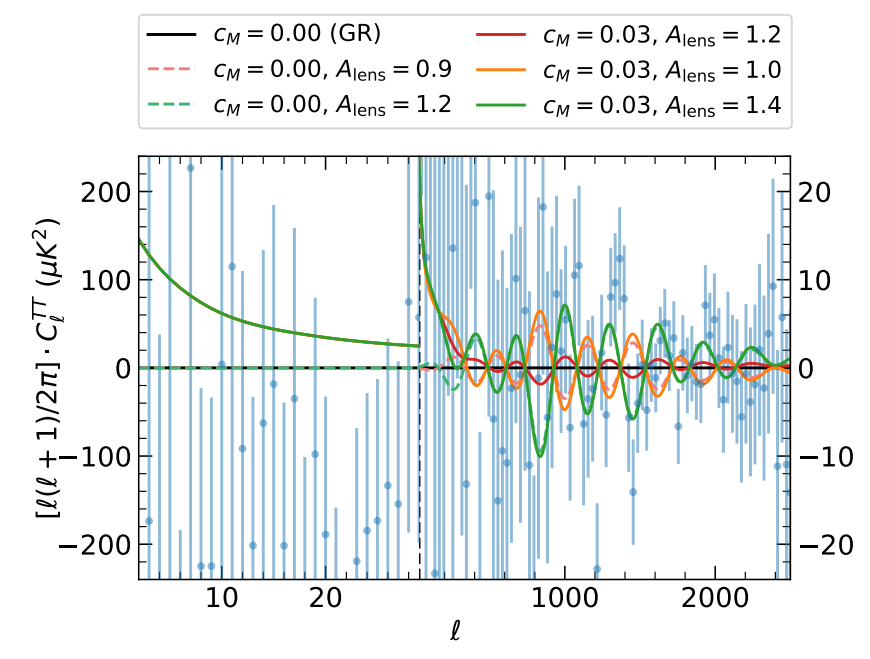

FIG. 8. In comparing the No Slip Gravity model to the Planck 2015 data (points with error bars) the lensing suppression from $c_{M}>0$ gives a worse fit than GR. However if we allow $A_{\text {lens }}>1$ then $c_{M}>0$ could be consistent in the acoustic oscillation region. We show three pairs of nearly matching curves of such a tradeoff. The fit at low $\ell$ remains discrepant (even with GR, though less so), though the origin of the low power in the data on large angular scales is unclear. Note the change from a linear scale in $\ell=[2,30]$ to log scale in $\ell>30$, and a change in the vertical scale as well.

- but the scale dependence means we cannot satisfy this simultaneously at both low and high multipoles. Figure 8 shows the residuals in TT power relative to $\Lambda$ CDM plus general relativity for three pairs of parameters. Increasing $c_{M}$ and $A_{\text {lens }}$ simultaneously can give nearly the same agreement with data in the acoustic peak region. This erases the $\ell>30$ penalty in $\chi^{2}$ from $c_{M}>0$, but leaves the penalty from $\ell<30$. The $95 \%$ confidence upper limit on $c_{M}$ remains about the same at 0.014 .

We explore the parameter constraints with MCMCs, both fixing $A_{\text {lens }}=1$ and allowing it to vary. When we fit for it, we obtain $A_{\text {lens }}=1.175 \pm 0.070$, consistent with Planck within general relativity. Since we do not find significant difference in the MCMC results when varying $A_{\text {lens }}$, for the figures (except Fig. 8 ) and tables we fix $A_{\text {lens }}=1$. The standard cosmological parameters are not significantly shifted from their concordance values, as seen in Table I. Figure 9 presents a triangle plot showing four of the parameters. We find that $c_{M}$ has almost no covariance with the other parameters due to its scale dependence.

The Planck data has $A_{\text {lens }}=1.180 \pm 0.065$ [20], as mentioned previously, while the Atacama Cosmology Telescope data gives $A_{\text {lens }}=1.06 \pm 0.15$ (stat) \pm 0.06 (sys) [24]. In the case $A_{\text {lens }}>1$ from some origin, $c_{M}>$ 0 suppresses the lensing back down and could give a good fit to $A_{\text {lens }}=1$. Conversely, the apparent $A_{\text {lens }}$ of, e.g., the POLARBEAR CMB experiment [19] with $A_{\text {lens }}=0.60_{-0.24}^{+0.26}$ (stat) ${ }_{-0.04}^{+0.00}$ (inst) \pm 0.14 (foreground) 


\begin{tabular}{c|c|c|c}
\hline Param & Mean $\pm \sigma$ & $95 \%$ lower & $95 \%$ upper \\
\hline $10^{2} \Omega_{b} h^{2}$ & $2.221_{-0.025}^{+0.025}$ & 2.170 & 2.274 \\
$\Omega_{\mathrm{cdm}} h^{2}$ & $0.1192_{-0.0022}^{+0.0022}$ & 0.1144 & 0.1238 \\
$H_{0}$ & $67.55_{-1.00}^{+1.03}$ & 65.44 & 69.84 \\
$\sigma_{8}$ & $0.8159_{-0.0096}^{+0.0117}$ & 0.7955 & 0.8373 \\
$c_{M}$ & $0.0053_{-0.0053}^{+0.0011}$ & 0.0000 & 0.0153
\end{tabular}

TABLE I. Results of the MCMC analysis with MontePython and hi_class for several parameters, keeping $A_{\text {lens }}=1$.

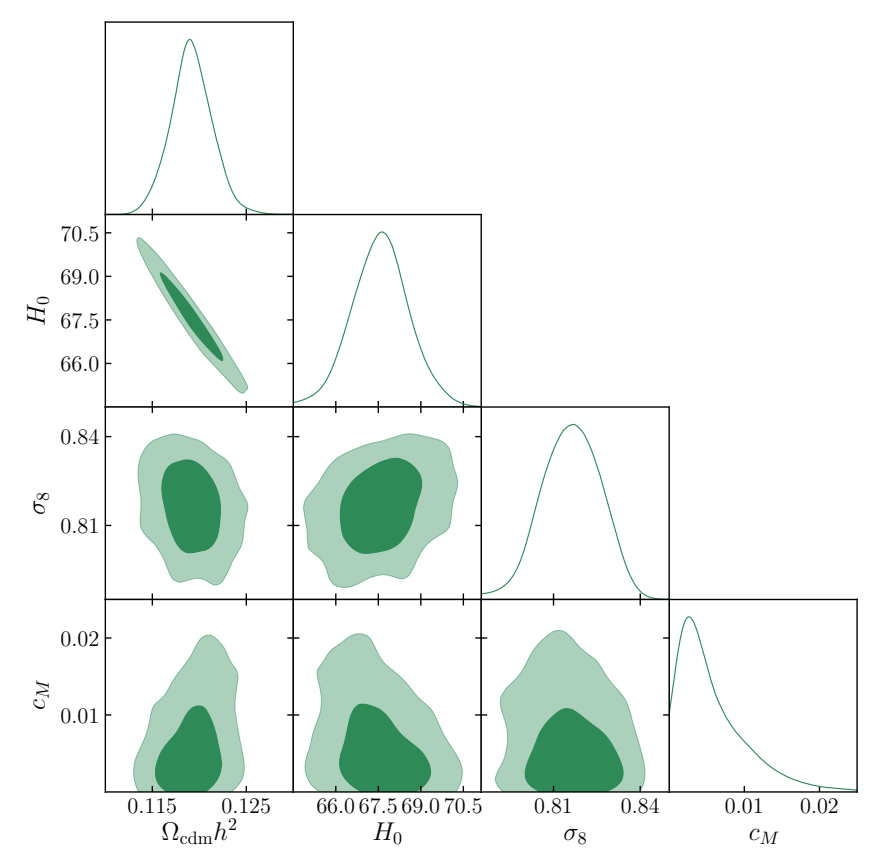

FIG. 9. The triangle plot for a few select parameters in the MCMC analysis with $A_{\text {lens }}=1$. We find that $c_{M}$ is preferred to be small, and is not covariant with other parameters, even if we allow $A_{\text {lens }}$ to vary.

\pm 0.04 (multi) or the South Pole Telescope with $A_{\text {lens }}=$ $0.81 \pm 0.14$ [25] could be interpreted as $A_{\text {lens }}=1$ with $c_{M}>0$.

Future, high accuracy polarization data, for example from the proposed CMB-S4 experiment, will play an important role in elucidating the hints of $A_{\text {lens }}$ deviations [26]. In addition, higher order CMB correlation functions and matter power spectrum measurements will place constraints on $A_{\text {lens }}$, No Slip Gravity, and general scale dependent physics.

\section{CONCLUSIONS}

No Slip Gravity is similar to the well studied $f(R)$ gravity in that it involves only one free function of time in addition to the expansion history. However, while $f(R)$ has no effect on light propagation, No Slip Gravity does and in a manner firmly connected to its effect on matter growth. That is, there is no slip so $G_{\text {matter }}=G_{\text {light }}$. Furthermore, due to the strength of its braiding No Slip Gravity can suppress structure growth while most scalartensor theories enhance it. These properties make it an attractive theory to study.

We focus on the effects on the CMB, in particular the primordial gravitational wave contribution to the $\mathrm{B}$ mode polarization as well as the lensing B modes. Implementing No Slip Gravity in the Boltzmann code hi_class (with the modification made publicly available on github at the URL given in Sec. II), we compute the CMB and matter density power spectra. Along the way we show that treatment of the kineticity $\alpha_{K}$ requires some consideration, and we present two ways for successfully treating it.

The CMB $\mathrm{B}$ mode reionization and recombination bumps from primordial gravitational waves are affected modestly in No Slip Gravity relative to general relativity, despite the equivalence of the gravitational wave speed of propagation, due to the running of the Planck mass $\alpha_{M}$. We find a fractional change to the tensor to scalar power ratio of $\delta r / r \lesssim 0.2 c_{M}$, where $c_{M}$ is maximum value of $\alpha_{M}$. For a gravity model designed to provide current cosmic acceleration but restore to general relativity in the early universe, the main effect is on the reionization bump at low multipoles $\ell \lesssim 10$.

The impact on lensing $\mathrm{B}$ mode polarization at higher multipoles $\ell \gtrsim 100$ is much more significant. Here the deviations from general relativity can be of order $4 c_{M}$, or tens of percent. We also investigate the CMB lensing power spectrum $C_{\ell}^{\phi \phi}$ itself and find a pleasingly accurate simple analytic expression for the deviation, based on the integrated effect of $G_{\text {matter }}-1$ and the direct relation in No Slip Gravity of $G_{\text {matter }}$ with $\alpha_{M}$ or $M_{\star}$. We discuss how scale dependence enters in $C_{\ell}^{\phi \phi}$ and how it gets washed out in $C_{\ell}^{B B}$ due to the angular convolution. This also distinguishes the CMB lensing amplitude $A_{\text {lens }}$ from the $\mathrm{B}$ mode amplitude $A_{B B}$.

Finally, we perform a Monte Carlo analysis of our No Slip Gravity model compared to the Planck 2015 TT data. We find that this CMB data prefers $c_{M}$ to be smaller than the large scale structure data points to; this comes equally from the higher than $\Lambda \mathrm{CDM}$ plus general relativity lensing of the $\mathrm{CMB}\left(A_{\text {lens }}>1\right)$ and the low large scale power in the CMB. Allowing $A_{\text {lens }}>1$ phenomenologically opens up somewhat the parameter space $c_{M}>0$ though the scale dependence means this is not a perfect tradeoff. Future polarization data can shed more light on these issues.

Current data are merely suggestive of a suppression in matter density growth relative to the concordance model of general relativity with $\Lambda \mathrm{CDM}$, e.g. from redshift space distortions or weak lensing, so there is no significant evidence for (or against) No Slip Gravity. Next generation CMB experiments such as CMB-S4 [27] and LiteBIRD [28] have as a major goal the measurement of the $\mathrm{B}$ mode polarization power spectrum from the signatures of primordial gravitational waves at low multi- 
poles to CMB lensing at higher multipoles. The computations presented here enable such observations to be an important test of general relativity vs modified gravity. No Slip Gravity is highly predictive, and will also be constrained from future galaxy surveys measuring the growth of structure, i.e. $f \sigma_{8}(z)$ and weak lensing. In addition, the theory gives clear relations between standard siren and photon luminosity distances to the same redshift that will be tested very precisely by upcoming gravitational wave detectors [29].

\section{ACKNOWLEDGMENTS}

This work is supported in part by the Energetic Cosmos Laboratory and by the U.S. Department of Energy, Office of Science, Office of High Energy Physics, under Award DE-SC-0007867 and contract no. DE-AC0205CH11231. MZ is supported by the Marie SklodowskaCurie Global Fellowship Project NLO-CO. MB acknowledges the support of the Natural Sciences and Engineering Research Council of Canada (NSERC), [PGSD2517114-2018]. This research used resources of the National Energy Research Scientific Computing Center, a DOE Office of Science User Facility supported by the Office of Science of the U.S. Department of Energy under Contract No. DE-AC02-05CH11231.
[1] M.A. Troxel et al., Dark Energy Survey Year 1 Results: Cosmological Constraints from Cosmic Shear, arXiv: 1708.01538

[2] C. Chang et al., A Unified Analysis of Four Cosmic Shear Surveys, arXiv:1808.07335

[3] H. Hildebrandt et al., KiDS-450: Cosmological parameter constraints from tomographic weak gravitational lensing, MNRAS 465, 1454 (2017) [arXiv:1606.05338]

[4] A. Leauthaud et al., Lensing is Low: Cosmology, Galaxy Formation, or New Physics?, MNRAS 467, 3024 (2017) [arXiv:1611.08606]

[5] S. Alam et al., The clustering of galaxies in the completed SDSS-III Baryon Oscillation Spectroscopic Survey: cosmological analysis of the DR12 galaxy sample, MNRAS 470, 2617 (2017) [arXiv:1607.03155]

[6] E.V. Linder, No Slip Gravity, JCAP 1803, 005 (2018) [arXiv:1801.01503]

[7] E. Bellini and I. Sawicki, Maximal freedom at minimum cost: linear large-scale structure in general modifications of gravity, JCAP 1407, 050 (2014) [arXiv:1404.3713]

[8] M. Denissenya, E.V. Linder, Gravity's Islands: Parametrizing Horndeski Stability, JCAP accepted, arXiv: 1808.00013

[9] B.P. Abbott et al., Gravitational Waves and GammaRays from a Binary Neutron Star Merger: GW170817 and GRB 170817A, Astrophys. J. Lett. 848, L13 (2017) [arXiv:1710.05834]

[10] M. Zumalacárregui, E. Bellini, I. Sawicki, J. Lesgourgues and P. G. Ferreira, "hi_class: Horndeski in the Cosmic Linear Anisotropy Solving System," JCAP 1708, 019 (2017) doi:10.1088/1475-7516/2017/08/019 [arXiv:1605.06102] ; ww. hiclass-code.net

[11] D. Blas, J. Lesgourgues and T. Tram, The Cosmic Linear Anisotropy Solving System (CLASS) II: Approximation schemes, JCAP 1107, 034 (2011) doi:10.1088/14757516/2011/07/034 [arXiv:1104.2933]

[12] D. Alonso, E. Bellini, P. G. Ferreira and M. Zumalacárregui, Observational future of cosmological scalar-tensor theories, Phys. Rev. D 95, 063502 (2017) doi:10.1103/PhysRevD.95.063502 [arXiv:1610.09290 [astro-ph.CO]].

[13] E. Bellini et al., Comparison of Einstein-Boltzmann solvers for testing general relativity, Phys. Rev.
D 97, 023520 (2018) doi:10.1103/PhysRevD.97.023520 [arXiv:1709.09135]

[14] I. D. Saltas, I. Sawicki, L. Amendola, M. Kunz, Anisotropic Stress as a Signature of Nonstandard Propagation of Gravitational Waves, Phys. Rev. Lett. 113, 191101 (2014) [arXiv:1406.7139]

[15] V. Pettorino, L. Amendola, Friction in Gravitational Waves: a test for early-time modified gravity, Phys. Lett. B 742, 353 (2015) [arXiv:1408.2224]

[16] A. Nishizawa, Generalized framework for testing gravity with gravitational-wave propagation. I. Formulation, Phys. Rev. D 97, 104037 (2018) [arXiv:1710.04825]

[17] S. Arai and A. Nishizawa, Generalized framework for testing gravity with gravitational-wave propagation. II. Constraints on Horndeski theory, Phys. Rev. D 97, 104038 (2108) [arXiv:1711.03776]

[18] M. Denissenya, E.V. Linder, Cosmic Growth Signatures of Modified Gravitational Strength, JCAP 1706, 030 (2017) [arXiv:1703.00917]

[19] POLARBEAR Collaboration, A Measurement of the Cosmic Microwave Background B-Mode Polarization Power Spectrum at Sub-Degree Scales from 2 years of POLARBEAR Data, ApJ 848, 141 (2017) [arXiv:1705.02907]

[20] Planck Collaboration, Planck 2018 results. VI. Cosmological parameters, arXiv:1807.06209

[21] B. Audren, J. Lesgourgues, K. Benabed and S. Prunet, Conservative Constraints on Early Cosmology: an illustration of the Monte Python cosmological parameter inference code, JCAP 1302, 001 (2013) doi:10.1088/14757516/2013/02/001 [arXiv:1210.7183]

[22] T. Brinckmann and J. Lesgourgues, MontePython 3: boosted MCMC sampler and other features, arXiv:1804.07261

[23] Planck Collaboration, Planck 2015 results. XI. CMB power spectra, likelihoods, and robustness of parameters, Astron. Astroph. 594, A11 (2016) [arXiv:1507.02704]; https://pla.esac.esa.int

[24] B.D. Sherwin et al., The Atacama Cosmology Telescope: Two-Season ACTPol Lensing Power Spectrum, Phys. Rev. D 95, 123529 (2017) [arXiv:1611.09753]

[25] J.W. Henning et al., Measurements of the Temperature and E-Mode Polarization of the CMB from 500 
Square Degrees of SPTpol Data, ApJ 852, 97 (2018) [arXiv:1707.09353]

[26] F. Renzi, E. Di Valentino, A. Melchiorri, Cornering the Planck $A_{\text {lens }}$ tension with future CMB data, Phys. Rev. D 97, 123534 (2018) [arXiv:1712.08758]
[27] K.N. Abazajian et al., CMB-S4 Science Book, First Edition, arXiv:1610.02743

[28] Y. Sekimoto et al., Concept design of the LiteBIRD satellite for CMB B-mode polarization, SPIE 10698, 106981Y (2018) ; https://doi.org/10.1117/12.2313432

[29] J.M. Ezquiaga and M. Zumalacárregui, Dark Energy in light of Multi-Messenger Gravitational-Wave astronomy, arXiv:1807.09241 